\title{
Cell adhesion molecules regulate contractile ring-indepen- dent cytokinesis in Dictyostelium discoideum
}

\author{
Akira Nagasaki ${ }^{1}$, Masamitsu Kanada ${ }^{1,2}$, Taro QP Uyeda ${ }^{1,2,3}$ \\ ${ }^{1}$ Research Institute for Cell Engineering, National Institute of Advanced Industrial Science and Technology (AIST), Tsukuba, \\ Ibaraki 305-8562, Japan; ${ }^{2}$ Graduate School of Life and Environmental Sciences, University of Tsukuba, Tsukuba, Ibaraki 305- \\ 8572, Japan; ${ }^{3}$ Biomedicinal Information Research Center, National Institute of Advanced Industrial Science and Technology (AIST), \\ Koto, Tokyo 135-0064, Japan
}

To investigate the roles of substrate adhesion in cytokinesis, we established cell lines lacking paxillin (PAXB) or vinculin (VINA), and those expressing the respective GFP fusion proteins in Dictyostelium discoideum. As in mammalian cells, GFP-PAXB and GFP-VINA formed focal adhesion-like complexes on the cell bottom. paxB ${ }^{-}$cells in suspension grew normally, but on substrates, often failed to divide after regression of the furrow. The efficient cytokinesis of $\operatorname{paxB}^{-}$cells in suspension is not because of shear forces to assist abscission, as they divided normally in static suspension culture as well. Double knockout strains lacking $m h c A$, which codes for myosin II, and $\operatorname{paxB}$ or $\operatorname{vin} A \operatorname{displayed}$ more severe cytokinetic defects than each single knockout strain. In mitotic wild-type cells, GFP-PAXB was diffusely distributed on the basal membrane, but was strikingly condensed along the polar edges in mitotic $m h c A^{-}$cells. These results are consistent with our idea that Dictyostelium displays two forms of cytokinesis, one that is contractile ringdependent and adhesion-independent, and the other that is contractile ring-independent and adhesion-dependent, and that the latter requires PAXB and VINA. Furthermore, that paxB ${ }^{-}$cells fail to divide normally in the presence of substrate adhesion suggests that this adhesion molecule may play additional signaling roles.

Keywords: Dictyostelium, paxillin, vinculin, cytokinesis, cell shapes and cell motility

Cell Research (2009) 19:236-246. doi: 10.1038/cr.2008.318; published online 9 December 2008

\section{Introduction}

In animal cells, mitotic cell division involves a highly coordinated series of events that lead to the formation of two daughter cells. It has been observed in many types of cells that actin and myosin II assemble to form a ring around the equatorial cortex during cytokinesis $[1,2]$. As actin and myosin II localize to the equatorial cortex during this process and as myosin II is the molecular motor that slides on actin filaments, it has been believed that active contraction of the ring powered by the activity of actin and myosin II drives the furrowing, and consequently cytokinesis. Interestingly, however, $m h c A$-null cells of the cellular slime mold Dictyostelium discoideum are

Correspondence: Akira Nagasaki

Tel: +81-29-861-3048; Fax: +81-29-861-3049

E-mail: a-nagasaki@aist.go.jp

Received 12 March 2008; revised 12 June 2008; accepted 30 June 2008; published online 9 December 2008 able to divide efficiently when they adhere to substrates apparently by making use of traction forces, which move the daughter cells away from one another [3-5]. Based on these observations, we and others have previously proposed that Dictyostelium has two major, mechanically distinct methods of cell cycle-coupled cytokinesis [3-7]. 'Cytokinesis A' $[4,5]$ is contractile ring (and thus myosin II)-dependent and substrate adhesion-independent, and is driven by active constriction of an equatorial cleavage furrow. 'Cytokinesis B' $[4,5]$, or attachment-assisted mitotic cleavage [3], on the other hand, is contractile ringindependent and substrate adhesion-dependent and makes use of traction forces to separate the daughter cells from one another. This model is not limited to Dictyostelium, as certain adherent mammalian cells are also able to divide by cytokinesis B when myosin II is inhibited [8].

Cytokinesis B does not require myosin II, but apparently is dependent on regulated adhesion to the substrate that is coordinated with traction forces. Furthermore, although cytokinesis A and B perform partially redundant 
functions, these two forms of cytokinesis must be regulated by at least partially distinct pathways. Thus, to investigate the mechanism of cytokinesis $\mathrm{B}$ or the contractile ring-independent and substrate adhesion-dependent cytokinesis, we decided to focus our research on the role of cell adhesion molecules in cytokinesis.

In vertebrate cells, substrate adhesion and cell migration are regulated through interactions between ligands derived from the extracellular matrix and transmembrane integrin molecules $[9,10]$. These points of interaction, which are called focal adhesions, associate with cytoplasmic actin filaments and play important roles in the regulation of the actin cytoskeleton. Among the other proteins that reportedly associate with focal adhesions is paxillin, which was originally identified as a substrate of the oncogenic tyrosine kinase v-src [11] and is thought to be an adapter protein that links the cytoplasmic domains of integrins to the actin cytoskeleton via vinculin $[12,13]$, thereby facilitating signal transduction from the extracellular matrix to the cell interior [14, 15]. Vinculin is a bipartite protein with the head and tail domains harboring binding sites for a number of proteins and lipids, and is also implicated in the formation of a cell adhesion complex [16]. The head domain of each vinculin molecule binds to talin, $\alpha$-actinin and intramolecularly to the tail domain, whereas the tail domain has binding sites for paxillin, actin and phosphatidylinositol 4,5-bisphosphate, as well as for the head within the same molecule [17].

Dictyostelium discoideum is an amoebic model organism with a simple genome and structure; yet its modes and mechanisms of motility and cytokinesis are very similar to those of neutrophils [18]. In addition, Dictyostelium shares many of the components involved in cell motility with mammalian cells $[19,20]$. Thus, a paxillinlike gene, $\operatorname{pax} B$, has been identified in the Dictyostelium genome, and recently Bukharova [21] reported the cloning and characterization of this gene. Furthermore, we found the gene encoding a vinculin-like protein in the Dictyostelium database and named it vinA. Here, we describe the phenotypes of $\operatorname{pax}^{-}, \operatorname{vin} A^{-}, \operatorname{pax}^{-} / m h c A^{-}$and $v i n A^{-} / m h c A^{-}$cells. The results support our cytokinesis A/ cytokinesis B model and, furthermore, suggest that cell adhesion molecules play important roles in cytokinesis B.

\section{Results}

\section{Cloning the Dictyostelium PAXB and VINA genes} paxB cDNA was amplified from a Dictyostelium cDNA library, and the sequence was confirmed to be identical to the published one [21]. Despite its name, paxB is the only PAXBlike gene found in the Dictyostelium genome sequence.

We found two genes, DDB0232320 and DDB0232319, each coding for a vinculin-like protein, in DictyBase (http://dictybase.org/). DDB0232320, named vinA, codes for an 842 amino acid protein with several segments that are highly similar to human vinculin (overall similarity is $18 \%$ and the average similarity of those homologous regions is $25 \%$; Supplementary information, Figure S1). In mammalian vinculin, these homologous regions contain binding sites for $\alpha$-actinin, talin, paxillin and actin, but we were unable to identify proline-rich binding sites for VASP and vinexin [22] (Supplementary information, Figure S1A, colored characters). The DDB0232319 gene codes for a 1748 amino acid protein, which is much larger than human vinculin (Supplementary information, Figure S1B); hence we did not further analyze this gene in this work.

\section{Expression of paxB and vinA}

To determine when paxB and vinA are expressed during the life cycle of $D$. discoideum, we isolated total RNA at various stages of the developmental program that leads to the formation of fruiting bodies and subjected them to RT-PCR analysis. As shown in Figure 1, the $\operatorname{pax} B$ and $\operatorname{vin} A$ transcripts were expressed at all stages in wild-type and $m h c A^{-}$cells, increasing with development and peaking at $12-18 \mathrm{~h}$ after the onset of starvation. Mitochondrial rRNA ig7, which served as an internal control, was expressed at a constant level during the developmental and vegetative stages.

\section{TIRF microscopy observation of GFP-PAXB and GFP- VINA}

To observe the intracellular localization of PAXB and VINA, we transformed wild-type and $m h c A^{-}$cells using an extrachromosomal expression vector, $\mathrm{pBIG}$, harboring the fusion gene $g f p$-pax $B$ or $g f p$-vinA under the control of the constitutive actin 15 promoter.

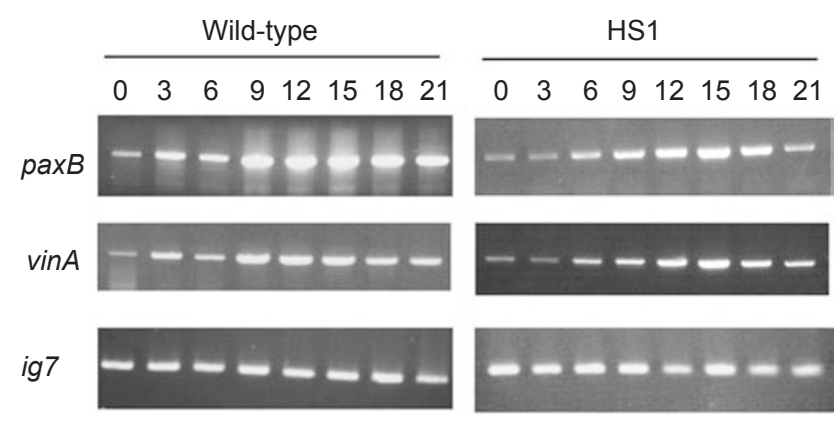

Figure 1 Expression of paxB and vinA during the life cycle of Dictyostelium. Cells were allowed to develop on non-nutritional agar for the times indicated, after which the DNA was amplified using the same amount of template RNA at each time point. Amplification of ig7 served as an internal control [47]. 
Distinct localization of GFP-PAXB and GFP-VINA in living cells was not observed by conventional fluorescent microscopy, owing to high background fluorescence. Thus, we used total internal reflection fluorescence (TIRF) microscopy. During interphase, GFP-PAXB localized to focal adhesions on the basal surface and at the tips of filopodia in wild-type and $m h c A^{-}$cells (Figure 2A), as reported by Bukharova et al. [21]. We could not find a significant difference in the distribution between interphase wild-type and $m h c A^{-}$cells. A representative example of wild-type and $m h c A^{-}$cells expressing GFP-PAXB undergoing cytokinesis on glass surfaces is shown in the right panels of Figure 2A. In mitotic wild-type cells, GFP-PAXB was present at the tips of filopodia, as well as over the entire cell membrane of the basal surface. In mitotic $m h c A^{-}$cells, in contrast, fluorescent dots of GFPPAXB were seen not only over the bottom membrane but also much more abundantly along the polar edges, including at the tips of protrusions. To investigate the difference of PAXB localization in more detail we used the agarose overlay method [23], by which cells were flattened. There were no differences between wild-type and $m h c A^{-}$cells in interphase, which showed scattered
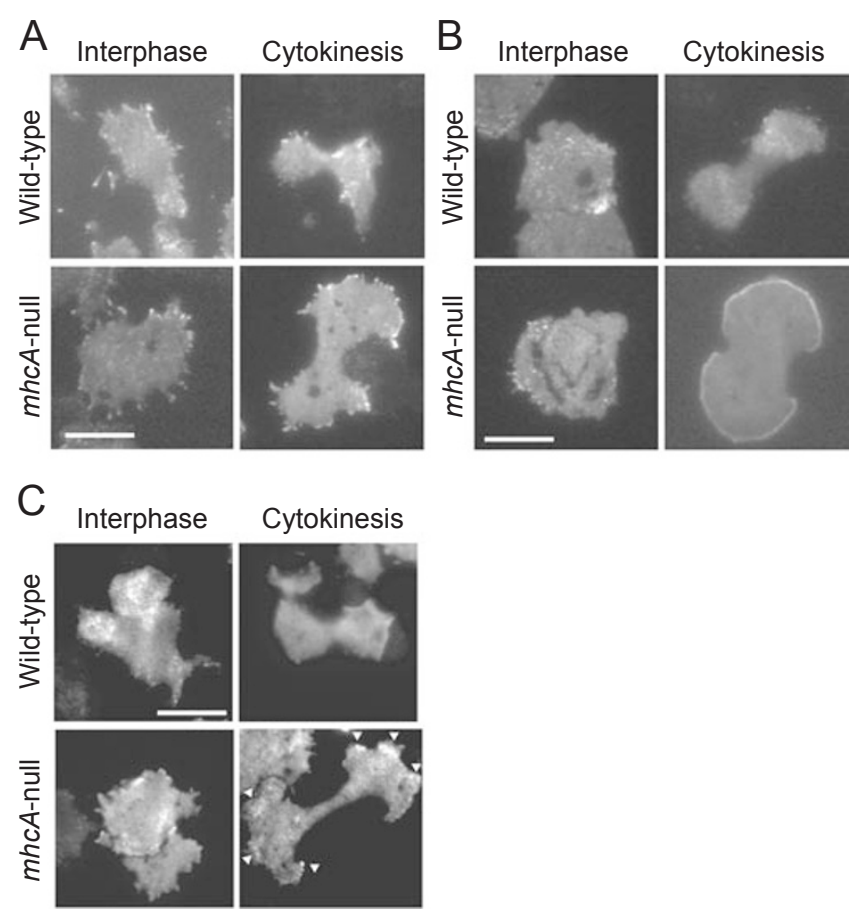

Figure 2 Subcellular localization of GFP-PAXB. TIRF images of the bottom of cells expressing GFP-PAXB under normal culture condition (A) and under thin agarose sheets (B). (C) Subcellular localization of GFP-VINA. TIRF images of the bottom of cells expressing GFP-VINA in normal culture condition. Bar: $10 \mu \mathrm{m}$. fluorescent dots of GFP-PAXB over the entire basal surface of the cells (Figure 2B). As wild-type cells entered mitosis, both the size and fluorescence intensity of the GFP-PAXB dots decreased, although the uniform distribution of the dots over the basal surface did not change. Localization of GFP-PAXB in mitotic $m h c A^{-}$cells was dramatically changed by the agarose overlay in that those cells showed a striking concentration of GFP-PAXB along the edges around both poles (Figure 2B).

Observation by TIRF microscopy permitted recognition of distinct localization of GFP-VINA as well (Figure $2 \mathrm{C})$. Clusters of small dots of GFP-VINA were found on the basal surface of the wild-type and $m h c A^{-}$cells during interphase. This distribution is similar to that of vinculin in human neutrophils [24]. When wild-type cells entered the mitotic phase, the number of fluorescent dots decreased on the cell bottoms. In contrast, dispersed fluorescence dots were observed over the whole basal surface of mitotic $m h c A^{-}$cells as in the interphase cells. Furthermore, intense localization of GFP-VINA was observed along the polar edges (Figure 2C, white arrowheads). These results suggest that localization of VINA to the basal surface is related to efficient cytokinesis $\mathrm{B}$, but not so for cytokinesis A. We next attempted to observe the localization of GFP-VINA by the agarose overlay method. For some unknown reason, however, fluorescent dots were no longer observed and fluorescence of GFPVINA was uniformly distributed over the whole cell bottom.

In addition, we observed MiDAS (mitosis-specific dynamic actin structure)-like structures in some mitotic $m h c A^{-}$cells expressing GFP-PAXB or GFP-VINA. MiDAS is an actin-containing cytoskeletal structure that was originally found underneath the nuclei in mitotic $m h c A^{-}$cells [25]. However, these structures were not apparent in all mitotic cells, and even when they are noticeable the enrichment of GFP-PAXB or GFP-VINA in the structures was weak and unclear when compared with that of actin reported by Itoh and Yumura (Supplementary information, Figure S2).

\section{PAXB-null cells exhibit impaired cytokinesis, motility and adhesion}

To explore the functions of PAXB and VINA in Dictyostelium cells, we generated cell lines lacking the $p a x B$ or vinA gene. The paxB or vinA loci were disrupted by homologous recombination with targeting constructs (Supplementary information, Figure S3A and S3C), and disruption was confirmed by genomic PCR using primers indicated by the arrows in Supplementary information, Figure S3A and S3C, respectively. In both cases, the PCR products were larger by $1.1 \mathrm{~kb}$, which corresponds 
to the size of the inserted marker gene (Supplementary information, Figure S3B and S3D).

As shown in Figures 3A and 4A, microscopic observation revealed that the sizes of $\operatorname{paxB}^{-}$cells were slightly larger than those of wild-type cells. The sizes of wildtype, pax $^{-}$cells and pax $B^{-}$cells expressing GFP-PAXB were $124.5 \pm 17.7,182.3 \pm 83.5$ and $123.3 \pm 45.6 \mu \mathrm{m}^{2}$, respectively $(n>20)$. The disruption of paxB also affected cell motility during both the vegetative and developmental stages (Figure 3B). During the vegetative stage, cells migrate in random directions by ameboid movement. When cells are transferred to a non-nutrient buffer, cells start the developmental process by moving toward aggregation centers. This process is mediated by chemotaxis toward cAMP, which orients the cells upward in the concentration gradient. We captured the images of cells every $10 \mathrm{~s}$ over a 30 -min period and calculated the cell speed using NIH Image, and found that the motil- ity of $\operatorname{pax}^{-}$cells was reduced to about $50 \%$ and $55 \%$ of control during the vegetative and developmental stages, respectively (Figure 3B).

As paxillin is required for normal substrate adhesion in mammalian cells [26], we next investigated whether substrate adhesion is impaired in paxB $B^{-}$Dictyostelium cells. For this purpose, we compared the time course of detachment of cells from substrates under continuous agitation. The adherent $\operatorname{pax}^{-}$cells began to detach from the substrate immediately after the onset of shaking, and by $60 \mathrm{~min}$ more than $90 \%$ of the $\mathrm{paxB}^{-}$cells were afloat in the medium (Figure 3C). By contrast, $67 \%$ of the wild-type cells remained adherent even after $60 \mathrm{~min}$ of shaking. Overexpression of GFP-fused PAXB was able to complement the defects of paxB $^{-}$cells (Figure $3 \mathrm{~B}$ and $3 \mathrm{C})$.

The larger size of paxB $^{-}$cells prompted us to stain their DNA with 4',6-diamidino-2-phenylindole (DAPI)

\section{A}

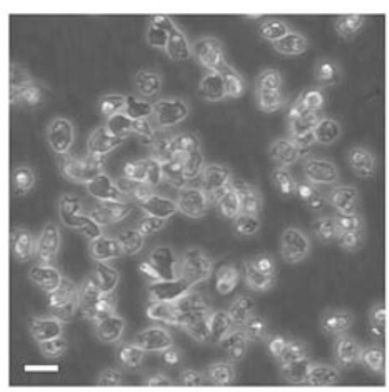

Wild-type

B

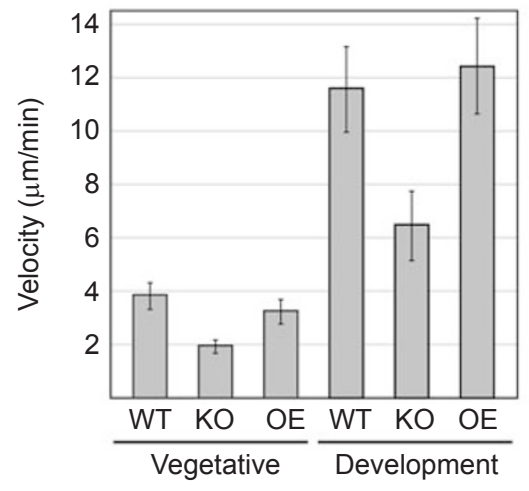

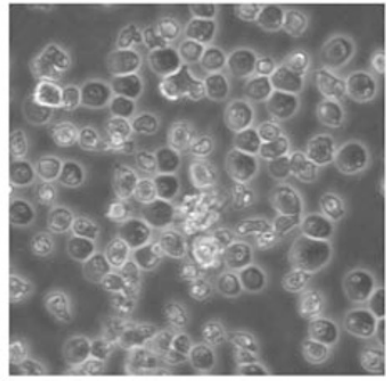

paxB-null

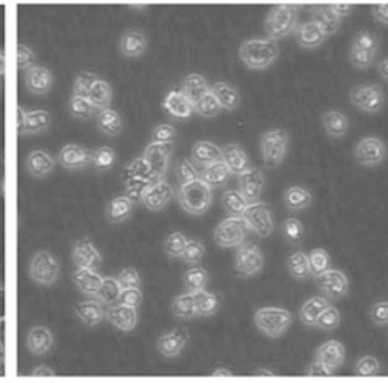

paxB OE

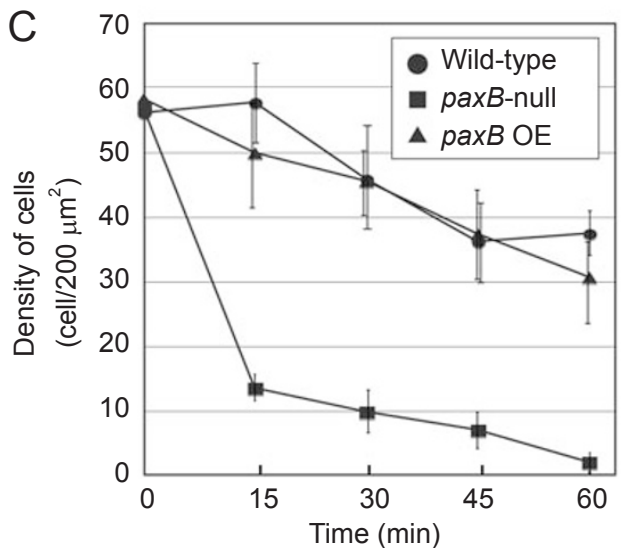

Figure 3 Phenotypes of paxB- cells. (A) Phase-contrast images of wild-type, paxB ${ }^{-}$cells and GFP-PAXB-expressing cells. Bar: $10 \mu \mathrm{m}$. (B) Motility of vegetative and developmental cells. The speed of wild-type and paxB cells was measured in HL5 and after starvation in $17 \mathrm{mM}$ phosphate buffer for $14 \mathrm{~h}$. Bars depict means \pm standard deviations $(n>30)$. (C) Substrate adhesion assay. The cells were allowed to grow on polystyrene dishes in HL5 medium for 1 day, and then shaken on a reciprocal shaker (speed: $110 \mathrm{rpm}$, amplitude: $3 \mathrm{~cm}$ ). The numbers of cells that remained adhered to the substrate were counted at the indicated times $(200 \mu \mathrm{m}$ squares from 10 plates each, means \pm standard deviations $)$. OE indicates the pax $B^{-}$cell line overexpressing GFP-PAXB. 
A

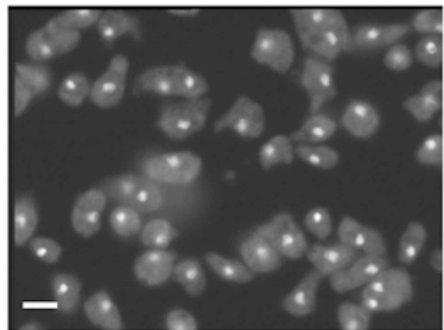

Wild-type

B

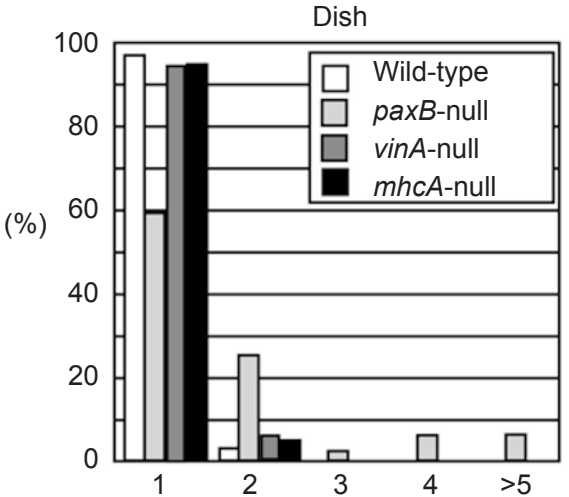

\section{(1)}

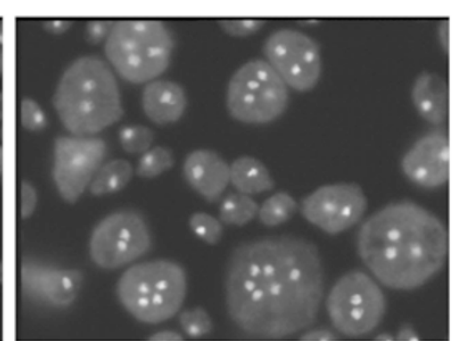

paxB-null

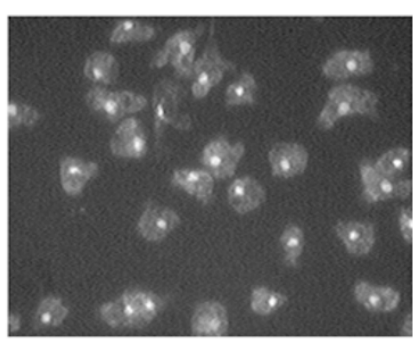

vinA-null for 3 days, fixed and stained with DAPI. Bar: $10 \mu \mathrm{m}$. (B) Distribution of the nuclei numbers per cell in wild-type, $m$ hc $A^{-}$, pax $B^{-}$ and vin $A^{-}$cells under three different culture conditions $(n>100)$. Histogram showing the percentage distribution of number of nuclei in wild-type (white), $m h c A^{-}$(black), $\operatorname{paxB}^{-}$(light gray) and vinA (dark gray) cells, cultivated on dishes, in suspension or on submerged agar surfaces.

to investigate the number of nuclei per cell (Figure 4A). We found that whereas $>90 \%$ of wild-type and $v i n A^{-}$ cells maintained on polystyrene substrates were mononucleate, about $40 \%$ of pax $^{-}$cells contained two or more nuclei (Figure 4B, left). On the other hand, when the cells were cultured in suspension, there were no significant differences in the numbers of nuclei per cell between wild-type and $p_{a x B^{-}}$cells, whereas $m h c A^{-}$cells became highly multinucleate (Figure $4 \mathrm{~B}$, center). Thus, fewer wild-type cells were multinucleate on substrates than in suspension ( $4 \%$ vs. $20 \%$ ), but fewer paxB $B^{-}$cells were multinucleate in suspension than on substrates $(22 \%$ vs. $41 \%$ ); moreover, that difference was even more striking when the numbers of highly multinucleate $(\geq 3$ nuclei per cell) pax $^{-}$cells were compared $(19 \%$ on substrates vs. $1 \%$ in suspension) (Figure 4B).

We also examined the cytokinesis of $\operatorname{paxB}^{-}$cells on the surface of $1.0 \%$ agar sheets immersed in HL-5 medium. Under this condition, $m h c A^{-}$cells, which require adhesion to a solid surface for cytokinesis, became large and multinucleate within 3-4 days as in suspension cul- ture (Figure 4B). By contrast, in the case of both wildtype and $\operatorname{paxB}^{-}$cells, cytokinesis was completed in most mitotic cells (Figure 4B).

\section{Time-lapse analysis of cytokinesis in pax $B^{-}$cells}

To investigate why $\operatorname{pax}^{-}$cells are less efficient at cytokinesis on solid surfaces, we performed a time-lapse observation of cytokinesis in paxB $^{-}$cells. Wild-type cells maintained on glass substrates completed cytokinesis within 180-240 $\mathrm{s}$ after the beginning of equatorial furrowing (Figure 5A). Mitotic paxB $B^{-}$cells also rounded up and displayed equatorial furrowing within $120-240 \mathrm{~s}$, but they often failed to complete cytokinesis and finally the dumbbell-shaped cells with deep furrows became single binucleate cells (Figure 5B and 5C). Furthermore, mitotic $\operatorname{pax}^{-}$cells tended to detach from the substrate more frequently than wild-type cells and to form larger protrusions that seemed to interfere with the normal progression of cytokinesis. For instance, one of the presumptive daughter cells in both Figure 5B and 5C seemed to detach from the substrate for a few minutes from $300 \mathrm{~s}$ 
A
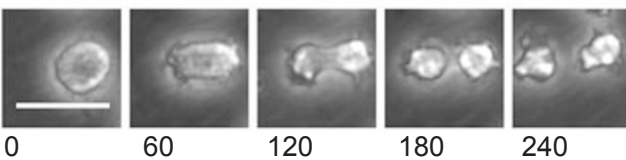

B
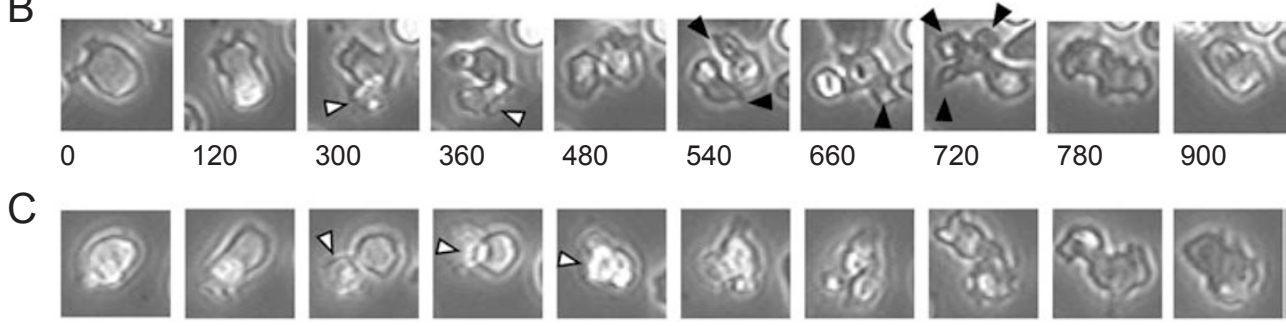

780

900

D
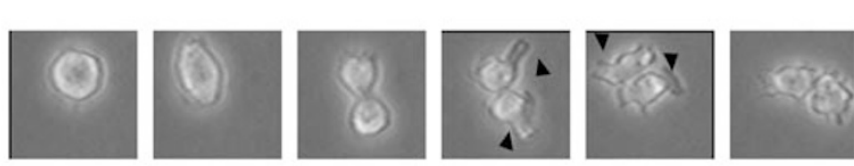

$0 \quad 60$

120

180

240

300

Figure 5 Sequences showing cytokinesis in mitotic wild-type and paxB ${ }^{-}$cells cultured on solid substrates. Each panel shows a series of phase-contrast images recorded at times indicated at the bottom left (seconds). (A) The wild-type cell completed cytokinesis within 2-3 min after initiation of equatorial furrowing. (B, C) Two cases of failed cytokinesis in pax $B^{-}$cells. Deep furrowing was observed in the equatorial region at $300 \mathrm{~s}(\mathbf{B}, \mathbf{C})$, but the furrows regressed and the daughter cells eventually merged into single cells containing two nuclei. (D) Wild-type cells were cultured on agar-coated substrate in medium. Under this condition, wild-type cells divide using cytokinesis A. White and black arrowheads show detached presumptive daughter cells and large protrusions, respectively. Bar: $20 \mu \mathrm{m}$.

Table 1 Cytokinesis phenotype in wild-type and paxB-null cells

\begin{tabular}{|c|c|c|c|c|c|}
\hline \multirow[t]{2}{*}{ Strain } & \multicolumn{2}{|l|}{ Cytokinesis } & \multicolumn{2}{|l|}{ Phenotype } & \multirow[t]{2}{*}{$N$} \\
\hline & success $(\%)$ & Failure $(\%)$ & prutrusion $^{1}(\%)$ & Detachment $^{2}(\%)$ & \\
\hline Wild-type & 100 & 0 & 29 & 17 & 24 \\
\hline
\end{tabular}

${ }^{1}$ Fraction of cells that formed large protrusions $(>2 \mu \mathrm{m})$ during cytokinesis.

${ }^{2}$ Fraction of mitotic cells of which at least one of the daughter cells detached from substrates during cytokinesis.

(white arrowheads), and both of the presumptive daughter cells in Figure 5B formed large protrusions around each pole at 540-720 s (black arrowheads). The length of large protrusions in mitotic wild-type and $\operatorname{pax}^{-}$cells that formed them during furrowing was $1.31 \pm 0.5(n=11)$ and $4.16 \pm 1.3 \mu \mathrm{m}(n=15)$, respectively. Quantitation of the cytokinesis phenotype of cells on substrates showed that all wild-type cells divided normally but $26 \%$ of mitotic pax $B^{-}$cells failed to complete cytokinesis (Table 1). This analysis also showed that a larger number of $\operatorname{paxB}^{-}$cells detached from the substrate and/or formed large protrusions. Furthermore, the abnormal shape and behavior of mitotic paxB $B^{-}$cells on solid surfaces were similar to those of wild-type cells on agar-coated substrates (Figure $5 \mathrm{D})$.
On the other hand, we could not find any morphological differences during cytokinesis between $\operatorname{vin} A^{-}$and wild-type cells on plastic substrates (data not shown). This result is consistent with that of nuclear staining with DAPI (Figure 4A, right).

\section{Knockout of mhcA in pax $B^{-}$or vinA $A^{-}$cells}

The findings summarized thus far suggest that $\operatorname{pax} B$ is involved in cytokinesis $\mathrm{B}$, so that $\operatorname{pax}^{-}$cells lacking the $m h c A^{-}$gene, which is essential for cytokinesis $\mathrm{A}$, should be defective in both cytokinesis A and B and, consequently, should become very large and highly multinucleate. To test that idea, we transfected $\operatorname{pax}^{-}$cells with a targeting construct against the $m h c A$ locus, and through homologous recombination obtained two independent 
double knockout isolates (Figure 6A). The majority of the cells in both isolates had become very large within 3 days after replating on a new glass-bottomed dish (Figure $6 \mathrm{~B}$ ), and nuclear staining with DAPI revealed that double knockout cells were much more highly multinucleate and larger than either of the single knockout strains (Figure 6C).

vin $A / m h c A$ double knockout cells also became large and multinucleate on solid substrates, even though each single disruption of the $v i n A$ or $m h c A$ gene did not affect cytokinesis under this condition (Figure $6 \mathrm{E}$ and $6 \mathrm{~F}$ ). These results suggest that, similar to $\operatorname{pax} B$, vin $A$ plays an important role in cytokinesis $\mathrm{B}$, but is not essential for cytokinesis A.

\section{Localization of VINA during cytokinesis B in mammalian} cells

Finally, we examined the distribution of vinculin in mitotic normal rat kidney (NRK) cells that were derived from the kidney of a rat. NRK cells on collagen-coated substrates were able to complete division by cytokinesis $\mathrm{B}$ when cells were treated with the myosin II specific inhibitor, blebbistatin, in a manner similar to Dictyostelium $m h c A^{-}$cells [8]. Thus, we immunostained mitotic
NRK cells after fixation in the absence (cytokinesis A) or presence (cytokinesis B) of blebbistatin. Vinculin formed dots on the basal membrane during interphase (data not shown), but disappeared from the basal membrane in cells undergoing cytokinesis A (Figure 7A, top). In contrast to this, cells undergoing cytokinesis $\mathrm{B}$ had vinculin dots along the edges of polar regions and on the basal surface (Figure 7A, bottom).

\section{Discussion}

It is generally thought that cytokinesis in animal cells is driven by contraction of an equatorial contractile ring in a manner dependent on the interaction between actin and myosin II [1, 27, 28]. To be sure, this 'purse-string' model accounts for cytokinesis in a variety of animal cell types. However, a number of observations that cannot be explained by this model have been reported [8, 29-31]. Most strikingly, $m h c A$-null Dictyostelium cells, which lack the single myosin II heavy chain gene, are able to divide efficiently on substrates $[3,4]$. Furthermore, the morphological changes during cytokinesis of $m h c A^{-}$cells were obviously distinct from those of wild-type cells [5]. To explain these, we proposed that Dictyostelium cells
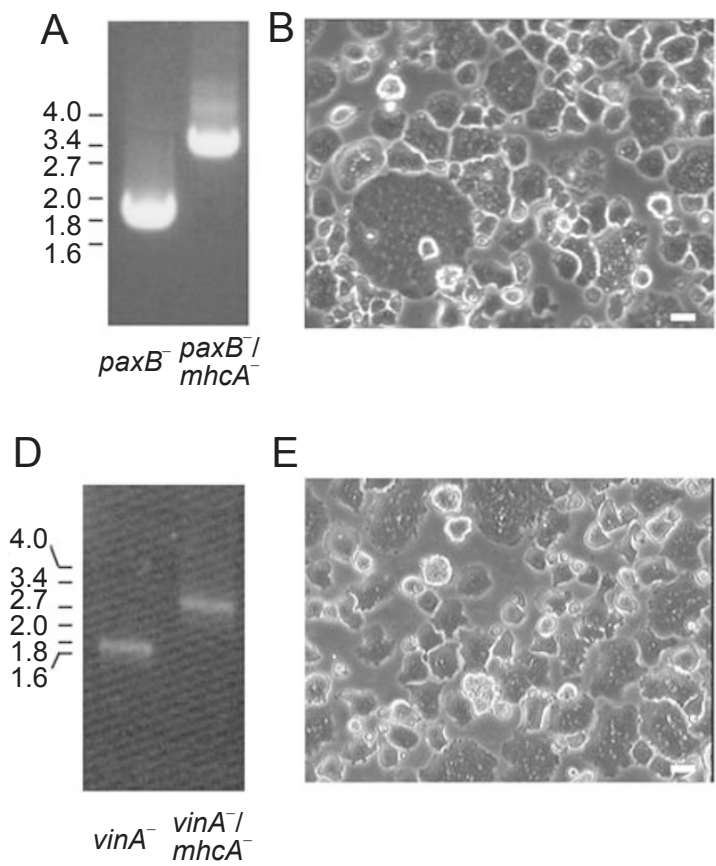

E

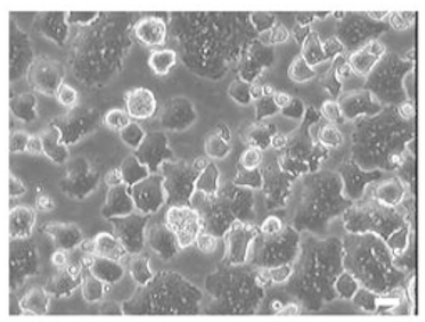

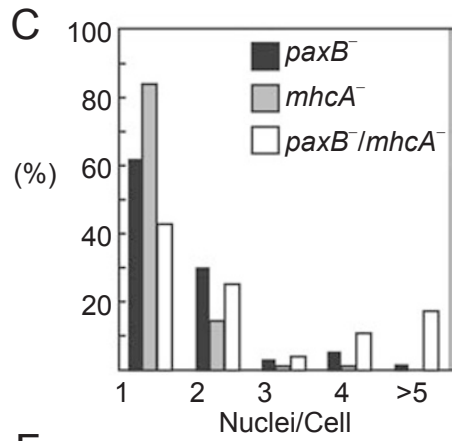

$\mathrm{F}$

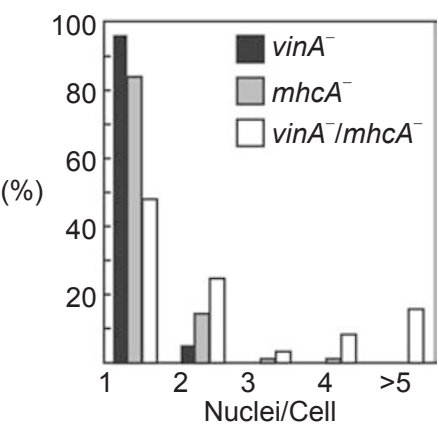

Figure 6 Disruption of the $m h c A$ gene in $\operatorname{pax}^{-}$and $v i n A^{-}$cells. (A, D) Mutant cells were identified by a shift in size of the PCR products. The targeting construct used to knockout $m h c A$ was described previously [5]. Phase-contrast micrographs of paxB $/ m h c A^{-}(\mathbf{B})$ and $v i n A^{-} / m h c A^{-}$double knockout cells (E). Cells were cultured on glass-bottomed dishes for 3 days. (C, F) Distribution of the numbers of nuclei per cell in the three cell lines $(n>100)$. Larger numbers of $p a x B^{-} / m h c A^{-}$cells and vinA $m h c A^{-}$cells were highly multinucleate than either single knockout cell line. Bar: $10 \mu \mathrm{m}$. 


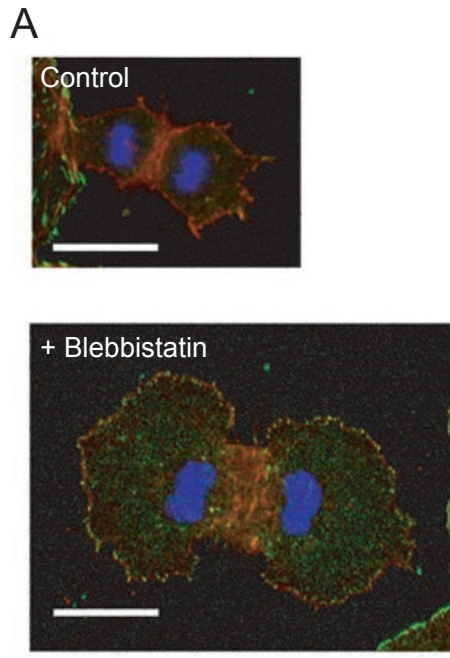

B

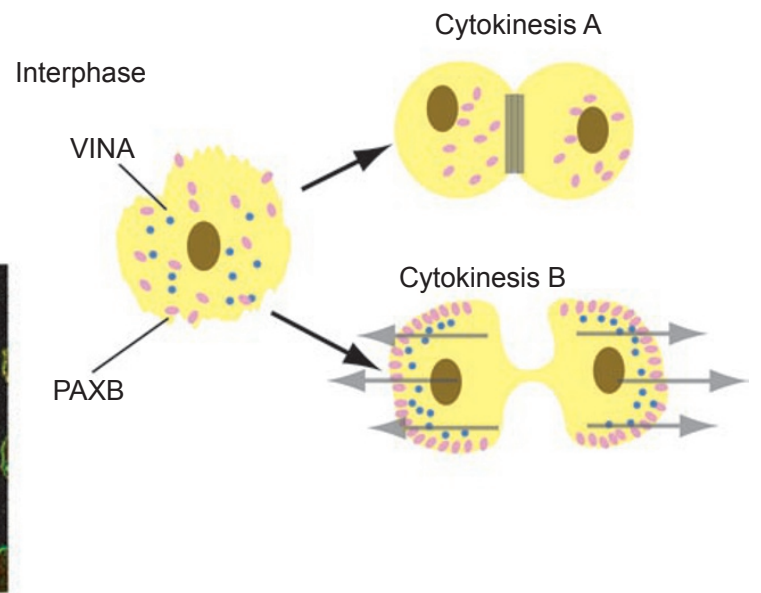

Figure 7 (A) Localization of vinculin in NRK cells undergoing cytokinesis A (control) or cytokinesis B (+blebbistatin). Cells were cultured on collagen-coated glass bottom dishes, fixed and stained with anti-vinculin antibody (green), Hoechst 33258 for DNA (blue) and rhodamine phalloidin for F-actin (red). Bar: $20 \mu \mathrm{m}$. (B) Schematic diagram illustrating the alteration of PAXB and VINA localizations. During interphase, PAXB and VINA localize over the whole cell bottoms and at the tips of filopodia to form a focal complex (left). When cell division is driven by contraction of the contractile rings (cytokinesis A), VINA disappears from the cell bottom and PAXB is distributed over the whole cell bottoms (upper right). In contrast, VINA does not disappear from cell bottoms and PAXB localizes along both polar peripheries during cytokinesis B (lower right).

have two distinct mechanisms of cytokinesis [5-7]: cytokinesis $\mathrm{A}$, which is dependent on active contraction of a contractile ring and is independent of substrate adhesion, and cytokinesis B [4, 5], or attachment-assisted mitotic cleavage [3], which does not depend on active contraction of a contractile ring but requires substrate adhesion. We have proposed that cytokinesis B is driven by oppositely oriented traction forces generated by two daughter cells, which separate the two cells by moving away from one another $[5,6]$, although a similar but somewhat different hypothesis has also been proposed $[32,33]$.

The aim of this study was to reveal the crosstalk among cell adhesion, cell migration and cytokinesis, cytokinesis B in particular, in Dictyostelium through examination of the functions of PAXB and VINA. Paxillin and vinculin are focal adhesion proteins that are conserved in a wide variety of eukaryotes including mammals, Xenopus and Drosophila [34]. Like the mammalian homologs [35], Dictyostelium PAXB and VINA localized at dotlike structures on the basal cell membrane facing the substrate, presumably representing focal adhesions (Figure 2).

pax $B^{-}$Dictyostelium cells showed a modest cytokinetic defect on substrates, and the cytokinetic defect was much more profound in $\mathrm{paxB}^{-} / m h c A^{-}$double knockout cells (Figure 6). These phenotypes suggest that PAXB plays an important role in cytokinesis B by facilitating the movement of daughter cells in opposite directions, because $m h c A^{-}$cells divide solely by cytokinesis B, which relies on substrate adhesion [3-5]. This speculation is supported by the fact that the migration speed and substrate adhesion of $\operatorname{pax}^{-}$cells were both reduced compared with those of the wild-type cells (Figure 3B and $3 \mathrm{C}$ ). It is further supported by the localization of GFP-PAXB in cytokinetic $m h c A^{-}$cells, at the tips of polar protrusions, or in the case of agarose overlay, along the edges around both poles. In contrast to this, there were fewer PAXB dots on the basal surface of wild-type cells undergoing cytokinesis. Thus, deletion of myosin II seems to somehow augment traction forces for completion of cytokinesis by recruiting PAXB along the polar peripheries, and it is of interest to investigate how this regulation is achieved at the molecular level.

Likewise, mitotic $m h c A^{-}$had small dots of GFP-VINA over the entire basal cell membrane, whereas in mitotic wild-type cells, the number of those dots decreased significantly and were observed only along polar edges on the basal membrane. Furthermore, vin $A^{-} / m h c A^{-}$double knockout cells became large and highly multinucleate (Figure 6), although a single knockout of the vinA gene did not noticeably affect cytokinesis (Figure 4). These results indicate that VINA is not required for cytokinesis A, but plays important roles in cytokinesis B, presumably by providing stronger substrate adhesion and larger 
traction forces. In addition, these results suggested that the localization of VINA during cytokinesis was also influenced by the lack of myosin II.

Nonetheless, impaired substrate adhesion and migration cannot explain why pax $B^{-}$cells exhibited more severe cytokinetic defects on substrates than in suspension (Figure 4). One obvious possibility why pax $^{-}$cells were able to divide more efficiently in suspension is that pax $B^{-}$cells have a general, modest cytokinetic defect regardless of substrate adhesion, but in shaking cultures, shear forces generated by agitation assisted the division. However, the fact that paxB $^{-}$cells were also able to divide and grow normally on immersed agar surfaces (Figure 4) showed that suspended $\operatorname{paxB}^{-}$cells did not divide by shear forces. One other intriguing and more likely possibility is that substrate adhesion normally elicits signal transductions that both positively and negatively regulate cytokinesis and cell migration, respectively, enabling highly coordinated movements, and that PAXB is involved in transmission of the positive signal. In this scenario, $\operatorname{paxB}^{-}$cells on substrates would receive only the negative signal, resulting in more frequent failure to divide than in suspension. Sun et al. [36] reported that $s p k A^{-}$cells also exhibited modest cytokinetic defects only when on substrates, and that the morphological abnormality of the mitotic $s p k A^{-}$cell is similar to that of the cytokinesis B mutants (A Nagasaki, unpublished data). This is particularly intriguing because we noticed that the domain structure of SAPKa, a novel stress-activated protein kinase that is the product of the $s p k A$ gene [36], is very similar to that of mammalian integrin-linked kinase (ILK), and it has been shown in mammalian cells that substrate adhesion receptors (e.g. integrins) mediate downstream signaling (outside-in signaling) when ligands bind to their extracellular domain [37]. Furthermore, the LD1 motif of paxillin, which binds directly to ILK in mammalian cells [38], is present in PAXB.

Our working model that summarizes the present data is shown in Figure 7B. In interphase Dictyostelium cells, PAXB and VINA localize to the cell bottom to form focal adhesion complexes and this distribution is not different between wild-type and $m h c A^{-}$cells. As cells initiate cytokinesis A, VINA disappears from the basal cell membrane but PAXB distributes over the entire basal cell membrane (Figure $7 \mathrm{~B}$, top right). On the other hand, the distribution of VINA and PAXB changes dramatically in response to the lack of myosin II. In mitotic $m h c A^{-}$cells, PAXB localizes along the polar peripheries of the two daughter cells, and VINA does not disappear from the basal cell membrane (Figure 7B, bottom right). Thus, the two adhesion molecules change their localizations depending on the conditions and the type of cytokinesis, and play particularly important roles in substrate adhesion and migration during cytokinesis B. Importantly, aspects of these regulatory mechanisms seem to be conserved between the cellular slime molds and mammalian cells, not only because of the structural similarities of the adhesion molecules but also from a functional point of view. For instance, certain adherent mammalian cells are capable of cytokinesis B when myosin II is inhibited, and divide in a manner similar to $m h c A^{-}$Dictyostelium cells [8]. Furthermore, loss of myosin II functions increased vinculin along polar edges and on the basal membrane during cytokinesis of both Dictyostelium and NRK cells (Figure 7). Thus, a deeper understanding of the mechanism of cytokinesis B in Dictyostelium should help us understand the mechanism of cytokinesis in mammalian cells as well. Intriguingly, a paxillin homolog, pxl1, was found to be incorporated into contractile rings of the fission yeast Schizosaccharomyces pombe, and depletion of plx 1 causes a delay in cell-cell separation $[39,40]$. It is however unclear how relevant these observations are to cytokinesis of animal cells as localization of paxillin is different between Dictyostelium and $S$. pombe, and, furthermore, cytokinesis of yeast cells is somewhat different in that it involves the formation of a septum.

\section{Materials and Methods}

\section{Cell culture}

Parental Dictyostelium discoideum wild-type AX2 and the HS1 $m h c A^{-}$cells [41] were grown axenically in HL-5 medium [42] supplemented with $6 \mu \mathrm{g} / \mathrm{ml}$ penicillin and streptomycin (Wako, Tokyo, Japan) at $21^{\circ} \mathrm{C}$. Each cell line lacking $\operatorname{paxB}$ or vinA was cultured in HL-5 in the presence of penicillin, streptomycin and 10 $\mu \mathrm{g} / \mathrm{ml}$ blasticidin S (Funakoshi, Tokyo, Japan). Double knockout cells, $\operatorname{paxB}^{-} / m h c A^{-}$and $v i n A^{-} / m h c A^{-}$, were cultured in the same medium described above containing both $10 \mu \mathrm{g} / \mathrm{ml}$ blasticidin $\mathrm{S}$ and $10 \mu \mathrm{g} / \mathrm{ml} \mathrm{G} 418$ (GIBCO). Cells carrying derivatives of the Dictyostelium expression vector pBIG [41] were grown in medium containing penicillin, streptomycin and $10 \mu \mathrm{g} / \mathrm{ml} \mathrm{G} 418$. The cells were usually grown on $9 \mathrm{~cm}$ plain polystyrene Petri dishes; in some experiments, however, they were grown in suspension, in conical Teflon flasks on a shaker rotating at $\sim 140 \mathrm{rpm}$.

\section{Molecular cloning of the paxB and vinA genes}

Full-length $\operatorname{pax} B$ and $\operatorname{vin} A$ cDNAs were cloned from a cDNA library of vegetative Dictyostelium Ax2 cells using RT-PCR. Components of the reverse transcription synthesis of the cDNA library included $1 \mu \mathrm{g}$ of poly(A) RNA, $1 \times$ reverse transcription buffer, $2 \mu \mathrm{M} d \mathrm{dTP}, 10 \mathrm{pmol}$ of QT primer, poly T primer (5'-CCA GTG AGC AGA GTG ACG AGG ACT CGA GCT CAA GCT TTT TTT TTT TTT TTT T-3' [43]) and $100 \mathrm{U}$ of reverse transcriptase (ReverTra Ace; TOYOBO, Osaka, Japan). The reaction mixture was incubated for $1 \mathrm{~h}$ at $42{ }^{\circ} \mathrm{C}$. The full-length cDNA of pax $B$ was amplified by PCR using a pair of primers 5'-GGATCC AAT GTC AAA TAA AAA TCC ATT AAA TAA TAG TA-3' and 5'-GAGCTC TTA TTT TCT TTG TTG TAC AAG TGT-3'. Prim- 
ers for amplification of vinA cDNA were 5'-GGATCC AAT GGA TGA AGT ATT AGA AAT GAT-3' and 5'-GAGCTC TTA TTG TTG TGG TAC TTT TCT-3'. These primers added BamHI and $\mathrm{SacI}$ recognition sites (underlined) at either end of the PCR products, enabling them to be subcloned into GFP/pBIG such that $p a x B$ or vinA cDNA was fused to the $3^{\prime}$ end of GFP cDNA in frame, the expression of which is driven by the actin 15 promoter [5].

\section{RT-PCR analysis}

AX2 and HS1 cells were allowed to develop on agarose plates containing $17 \mathrm{mM}$ phosphate buffer [44]. Using ISOGEN (Nippon Gene, Tokyo, Japan), total RNA was purified from the developing AX2 and HS1 cells at the indicated times, after which RT-PCR was carried out for 25 cycles with primers specific for $\operatorname{pax} B$ and $\operatorname{vin} A$.

Generation of paxB and vinA-null cells

paxB and vinA-null cells of Dictyostelium were generated by homologous recombination. Genomic DNA encoding pax $B$ and vinA was cloned into pGEM-T easy vector (Promega, Tokyo, Japan), and the blasticidin $\mathrm{S}$ resistance cassette from mtag (Bsr) [45] was inserted at the unique $B g / I I$ and $E c o$ RI site within the paxB and $\operatorname{vin} A$ genes, respectively, yielding the targeting vectors. Ten $\mu \mathrm{g}$ of $\operatorname{pax} B$ or vinA targeting vector was then linearized with $P v u \mathrm{II}$ and introduced into wild-type AX2 cells. Transformants were selected for blasticidin S resistance, and the colonies formed on plastic Petri dishes were isolated. Genomic PCR was then carried out to verify the disruption of the $\operatorname{paxB}$ and $\operatorname{vin} A$ genes. $p a x B^{-} / m h c A^{-}$ and $v i n A^{-} / m h c A^{-}$double knockout cells were generated from pax $B^{-}$or $v i n A^{-}$cells by using a targeting vector against the myosin II heavy chain gene (pKO myo(Neo)) [5].

\section{Fluorescence microscopy}

To investigate the localization of PAXB and VINA in living cells, AX2 and $m h c A^{-}$cells were transfected with $g f p$-paxB/pBIG or $g f p$-vinA/pBIG by electroporation, after which the resultant transfectants were transferred to plastic Petri dishes with thin glass bottoms (IWAKI, Funabashi, Japan). For the detailed observation of GFP-PAXB or GFP-VINA on the basal surface of the cells, we employed TIRF microscopy. The Olympus TIRF system was combined with an inverted microscope (IX71, Olympus, Tokyo, Japan) and a high-aperture objective lens (Apo 100× OHR; NA 1.65, Olympus) connected to an EB-CCD camera (C7190; Hamamatsu Photonics, Hamamatsu, Japan) that was operated with the NIHimage software. To observe GFP fusion proteins, we used a 488-nm laser.

NRK cells were cultured in DMEM (Sigma, Tokyo, Japan) supplemented with $10 \%$ fetal bovine serum (FBS) and 1\% antibioticantimycotic (Invitrogen, Tokyo, Japan) on collagen-coated glass bottom dishes with or without $30 \mu \mathrm{M}$ blebbistatin. For immunofluorescence staining, cells were fixed with $3.7 \%$ neutralized formaldehyde for $10 \mathrm{~min}$, permeabilized with acetone at $-20{ }^{\circ} \mathrm{C}$ for $5 \mathrm{~min}$, and stained with rhodamine-labeled phalloidin (Molecular Probes, Tokyo, Japan), anti-vinculin antibody (hVIN1, Sigma) and Hoechst 33258 (Wako).

\section{Cell motility and adhesion assays}

Quantitative analysis of the motility of cells in the growth phase and developmental phase was carried out essentially as described by Asano et al. [46] using an Olympus inverted microscope equipped with a Sony CCD camera (XC-ST50) and an image-processing system. Time-lapse images were acquired for $1 \mathrm{~h}$ with intervals of $10 \mathrm{~s}$ between each image, and the speed of the cell migration was calculated using ImageJ software.

To investigate the effect of PAXB deficiency on substrate adhesion, we developed a simple assay as follows. Cells were transferred to $60 \mathrm{~mm}$ plastic dishes (BD Falcon) containing $4 \mathrm{ml}$ of medium and incubated for $8 \mathrm{~h}$ to allow them to fully adhere to the substrate. The dishes were placed on a horizontal reciprocal shaker (Nippon Genetics, Tokyo, Japan) operating at 110 strokes/ min (amplitude, $30 \mathrm{~mm}$ ). Thereafter, the numbers of the cells that remained attached to substrates were counted in micrographs of randomly chosen $200 \mu \mathrm{m} \times 200 \mu \mathrm{m}$ areas taken at appropriate intervals with an $10 \times$ objective lens using an inverted microscope (Olympus).

\section{Acknowledgments}

We thank the Japan Society for the Promotion of Science for awarding a fellowship to AN.

\section{Reference}

1 Mabuchi I, Okuno M. The effect of myosin antibody on the division of starfish blastomeres. J Cell Biol 1977; 74:251-263.

2 Glotzer M. The molecular requirements for cytokinesis. Science 2005; 307:1735-1739.

3 Neujahr R, Heizer C, Gerisch G. Myosin II-independent processes in mitotic cells of Dictyostelium discoideum: redistribution of the nuclei, re-arrangement of the actin system and formation of the cleavage furrow. $J$ Cell Sci 1997; 110:123-137.

4 Zang JH, Cavet G, Sabry JH, et al. On the role of myosin-II in cytokinesis: division of Dictyostelium cells under adhesive and nonadhesive conditions. Mol Biol Cell 1997; 8:2617-2629.

5 Nagasaki A, de Hostos EL, Uyeda TQ. Genetic and morphological evidence for two parallel pathways of cell-cycle-coupled cytokinesis in Dictyostelium. J Cell Sci 2002; 115:22412251.

6 Nagasaki A, Hibi M, Asano Y, Uyeda TQ. Genetic approaches to dissect the mechanisms of two distinct pathways of cell cycle-coupled cytokinesis in Dictyostelium. Cell Struct Funct 2001; 26:585-591.

7 Uyeda TQ, Nagasaki A. Variations on a theme: the many modes of cytokinesis. Curr Opin Cell Biol 2004; 16:55-60.

8 Kanada M, Nagasaki A, Uyeda TQ. Adhesion-dependent and contractile ring-independent equatorial furrowing during cytokinesis in mammalian cells. Mol Biol Cell 2005; 16:38653872 .

9 Worthylake RA, Burridge K. Leukocyte transendothelial migration: orchestrating the underlying molecular machinery. Curr Opin Cell Biol 2001; 13:569-577.

10 Gumbiner BM. Cell adhesion: the molecular basis of tissue architecture and morphogenesis. Cell 1996; 84:345-357.

11 Glenney JR Jr, Zokas L. Novel tyrosine kinase substrates from Rous sarcoma virus-transformed cells are present in the membrane skeleton. J Cell Biol 1989; 108:2401-2408.

12 Schaller MD, Otey CA, Hildebrand JD, Parsons JT. Focal adhesion kinase and paxillin bind to peptides mimicking beta integrin cytoplasmic domains. J Cell Biol 1995; 130:1181-1187. 
13 Liu S, Thomas SM, Woodside DG, et al. Binding of paxillin to alpha4 integrins modifies integrin-dependent biological responses. Nature 1999; 402:676-681.

14 Turner CE. Paxillin interactions. J Cell Sci 2000; 113:413941413940.

15 Schaller MD. Paxillin: a focal adhesion-associated adaptor protein. Oncogene 2001; 20:6459-6472.

16 Critchley DR. Focal adhesions - the cytoskeletal connection. Curr Opin Cell Biol 2000; 12:133-139.

17 Ziegler WH, Liddington RC, Critchley DR. The structure and regulation of vinculin. Trends Cell Biol 2006; 16:453-460.

18 Egelhoff TT, Spudich JA. Molecular genetics of cell migration: Dictyostelium as a model system. Trends Genet 1991; 7:161-166.

19 Eichinger L, Pachebat JA, Glockner G, et al. The genome of the social amoeba Dictyostelium discoideum. Nature 2005; 435:43-57.

20 Kuspa A, Loomis WF. The genome of Dictyostelium discoideum. Methods Mol Biol 2006; 346:15-30.

21 Bukharova T, Weijer G, Bosgraaf L, et al. Paxillin is required for cell-substrate adhesion, cell sorting and slug migration during Dictyostelium development. J Cell Sci 2005; 118:4295-4310.

22 Zamir E, Geiger B. Molecular complexity and dynamics of cell-matrix adhesions. J Cell Sci 2001; 114:3583-3590.

23 Fukui Y, Yumura S, Yumura TK. Agar-overlay immunofluorescence: high-resolution studies of cytoskeletal components and their changes during chemotaxis. Methods Cell Biol 1987; 28:347-356.

24 Yuruker B, Niggli V. Alpha-actinin and vinculin in human neutrophils: reorganization during adhesion and relation to the actin network. J Cell Sci 1992; 101:403-414.

25 Itoh G, Yumura S. A novel mitosis-specific dynamic actin structure in Dictyostelium cells. J Cell Sci 2007; 120:4302-4309.

26 Brown MC, Perrotta JA, Turner CE. Serine and threonine phosphorylation of the paxillin LIM domains regulates paxillin focal adhesion localization and cell adhesion to fibronectin. Mol Biol Cell 1998; 9:1803-1816.

27 Glotzer M. The mechanism and control of cytokinesis. Curr Opin Cell Biol 1997; 9:815-823.

28 Robinson D, Spudich J. Towards a molecular understanding of cytokinesis. Trends Cell Biol 2000; 10:228-237.

29 O'Connell CB, Warner AK, Wang Y. Distinct roles of the equatorial and polar cortices in the cleavage of adherent cells. Curr Biol 2001; 11:702-707.

30 Tolliday N, Pitcher M, Li R. Direct evidence for a critical role of myosin II in budding yeast cytokinesis and the evolvability of new cytokinetic mechanisms in the absence of myosin II. Mol Biol Cell 2003; 14:798-809.

31 Urven LE, Yabe T, Pelegri F. A role for non-muscle myosin II function in furrow maturation in the early zebrafish embryo. $J$
Cell Sci 2006; 119:4342-4352.

32 Zhang W, Robinson DN. Balance of actively generated contractile and resistive forces controls cytokinesis dynamics. Proc Natl Acad Sci USA 2005; 102 :7186-7191.

33 Effler JC, Kee YS, Berk JM, et al. Mitosis-specific mechanosensing and contractile-protein redistribution control cell shape. Curr Biol 2006; 16:1962-1967.

34 Tumbarello DA, Brown MC, Turner CE. The paxillin LD motifs. FEBS Lett 2002; 513:114-118.

35 Salgia R, Li JL, Ewaniuk DS, et al. Expression of the focal adhesion protein paxillin in lung cancer and its relation to cell motility. Oncogene 1999; 18:67-77.

36 Sun B, Ma H, Firtel RA. Dictyostelium stress-activated protein kinase alpha, a novel stress-activated mitogen-activated protein kinase kinase kinase-like kinase, is important for the proper regulation of the cytoskeleton. Mol Biol Cell 2003; 14:4526-4540.

37 Liu S, Calderwood DA, Ginsberg MH. Integrin cytoplasmic domain-binding proteins. J Cell Sci 2000; 113:3563-3571.

38 Nikolopoulos SN, Turner CE. Molecular dissection of actopaxin-integrin-linked kinase-Paxillin interactions and their role in subcellular localization. J Biol Chem 2002; 277:1568-1575.

39 Ge W, Balasubramanian MK. Pxllp, a Paxillin-related protein, stabilizes the actomyosin ring during cytokinesis in fission yeast. Mol Biol Cell 2008; 19:1680-1692.

40 Pinar M, Coll PM, Rincon SA, Perez P. Schizosaccharomyces pombe Pxl1 is a paxillin homologue that modulates Rhol activity and participates in cytokinesis. Mol Biol Cell 2008; 19:1727-1738.

41 Ruppel KM, Uyeda TQ, Spudich JA. Role of highly conserved lysine 130 of myosin motor domain. In vivo and in vitro characterization of site specifically mutated myosin. $J$ Biol Chem 1994; 269:18773-18780.

42 Sussman M. Cultivation and synchronous morphogenesis of Dictyostelium under controlled experimental conditions. Methods Cell Biol 1987; 28:9-29.

43 Frohman MA. On beyond classic RACE (rapid amplification of cDNA ends). PCR Methods Appl 1994; 4:S40-S58.

44 Fukui Y. Actomyosin organization in mitotic Dictyostelium amoebae. Ann N Y Acad Sci 1990; 582:156-165.

45 Hibi M, Nagasaki A, Takahashi M, Yamagishi A, Uyeda TQ. Dictyostelium discoideum talin A is crucial for myosin IIindependent and adhesion-dependent cytokinesis. J Muscle Res Cell Motil 2004; 25:127-140.

46 Asano Y, Mizuno T, Kon T, et al. Keratocyte-like locomotion in amiB-null Dictyostelium cells. Cell Motil Cytoskeleton 2004; 59:17-27.

47 Chang WT, Newell PC, Gross JD. Identification of the cell fate gene stalky in Dictyostelium. Cell 1996; 87:471-481.

(Supplementary information is linked to the online version of the paper on the Cell Research website.) 